\title{
Non-Hodgkin lymphoma of sinuses and nasal cavity: case series of two
}

\section{Deepak Dalmia*, Sanjaya Kumar Behera, Jas Simran Singh Bhatia}

Department of Otorhinolaryngology, Dr. Babasaheb Ambedkar Central Railway Hospital, Mumbai, India

Received: 10 March 2017
Accepted: 18 April 2017

*Correspondence:

Dr. Deepak Dalmia,

E-mail: drjassimran@gmail.com

Copyright: () the author(s), publisher and licensee Medip Academy. This is an open-access article distributed under the terms of the Creative Commons Attribution Non-Commercial License, which permits unrestricted non-commercial use, distribution, and reproduction in any medium, provided the original work is properly cited.

\begin{abstract}
Non-Hodgkin lymphoma is a type of hematologic malignancy derived from lymphocytes, which varies significantly in their severity, from slow growing to very aggressive types. Incidence increases with age. Up to 45 years of age Non-Hodgkin lymphoma is more common among males than females. Various etiological factors includes infectious agents like Epstein-barr virus, Human T-cell leukemia virus, Helicobacter pylori, Human herpes virus-8, Hepatitis c virus, HIV etc. Exposure to chemical like polychlorinated biphenyls, phenoxy herbicides and medical treatments like radiation therapy and chemotherapy also act as etiological factors. Genetic diseases like Klinefelter's syndrome, ataxia telangiectasia and autoimmune diseases like celiac sprue, rheumatoid arthritis and systemic lupus erythematosus can also act as causal factors. NHL in nose and nasal cavity is a rare finding. Ours is a case series of two rare case of non-Hodgkin lymphoma presented with a nasal mass with nasal obstruction. The first case is a sixty years old female patient who presented with a right nasal mass with nasal obstruction. The second case is a fifty years old male who presented with left side nasal mass with left proptosis. We diagnosed these cases by taking biopsy from the nasal masses. These cases highlighted the importance of good clinical history and examination along with management according to definite protocols in regarding to avoid missing a rare.
\end{abstract}

Keywords: Lymphoma, Nasal cavity, Sinus

\section{INTRODUCTION}

Hematologic malignancies are very often seen in immune compromised patients. ${ }^{1}$ Lymphoma is the second most common primary malignancy occurring in the head and neck. Non-Hodgkin's lymphoma (NHL) is a group of neoplasms that originate from the cells of the lymphoreticular system. Forty percent of non-Hodgkin's lymphoma arises from extranodal sites. Common primary extranodal sites of lymphomas include stomach, liver, soft tissue, dura, bone, intestine and bone marrow. The nasal cavities and paranasal sinuses are rarely affected by primary NHL. ${ }^{2}$ Large B-cell lymphoma (LBCL) is the most common non-Hodgkin's lymphoma. LBCL is a fast growing malignancy that may arise inside or outside of the lymphatic system. Non-Hodgkin's lymphoma has a varied manner of presentation, response to therapy and prognosis. A few cases of oral lymphomas were reported in association with acquired immune deficiency syndrome (AIDS), and it might even be the first presentation of AIDS in certain individuals. Clinical presentations vary according to the histological type. The low grade lymphomas present with a nasal cavity or paranasal sinus mass associated with obstructive symptoms and/or lymphadenopathy, while high grade lymphomas present with aggressive signs and symptoms including non-healing ulcer, epistaxis, septal perforation and bone destruction. Lymphomas are usually submucosal, and on gross appearance, differ from SCC which is usually ulcerative. The vast majority of patients with localized 
disease are curable with combined modality therapy. About $50 \%$ patients are cured with doxorubicin based combination chemotherapy and rituximab.

\section{CASE REPORT}

\section{First case}

A 60 years old female patient presented in the OPD of ENT department with chief complaints of blockage in right side of nasal cavity, which was insidious in onset, gradually progressive in nature and was not associated with nasal discharge and anosmia. Patient also noticed a swelling in right side of nose arising from lateral wall of nose about one month ago. It was insidious in onset and gradually progressive in nature. There was no history of epistaxis, recurrent sneezing, itching and headache. The consent of the patient was taken before clinical examination and taking photograph. On examination, a diffuse swelling was present on the right side of the dorsum of the nose. No scar / sinuses seen. On anterior rhinoscopy, an irregular mass was seen in right nasal cavity. On probing the mass didn't bled, insensitive to pain, soft in consistency. Probe can't be passed all around the swelling. Air entry was decreased in right side on cold spatula test. On posterior rhinoscopy no growth was seen.

There was no lymphadenopathy, no limitation of eye movements or visual acuity. She was afebrile. Further systemic examination was unremarkable. Her $\mathrm{Hb}$ was $13.4 \mathrm{gm} \%$, total count was 8800, DLC was N68L28, BT was 1 '10", CT was 3'55", FBS was $87 \mathrm{mg} \%$, PPBS was $120 \mathrm{mg} \%$. Chest X-RAY was normal.

Radiological examination - CT PNS - Soft tissue mucosal thickening over right inferior turbinate causing almost complete occlusion of nasal cavity. Small left concha bullosa is noted with mild nasal septal deviation to left side (Figure 1 and 2).

Patient underwent right nasal mass biopsy and the specimen was sent for histopathological study. The histopathological examination reveals extra nodal nonHodgkin lymphoma, diffuse large B-cell type. Further patient underwent bone marrow aspiration and bone marrow biopsy which revealed normocellular uninvolved bone marrow. Patient also underwent microscopic examination of CSF which was negative for malignancy. On 2-D Echo, LVEF was 65\%. Computed tomography of the abdomen was normal. Patient received prephase chemotherapy of injection cyclophosphamide and intracthecal injection of methotrexate. Later patient received injection rituximab, injection vincristine, injection doxorubicin and injection cyclophosphamide after four days of receiving prephase chemotherapy. On fifth day patient received injection pepfilgrastim $(6 \mathrm{mg}$ subcutaneous).

\section{Prechemotherapy}

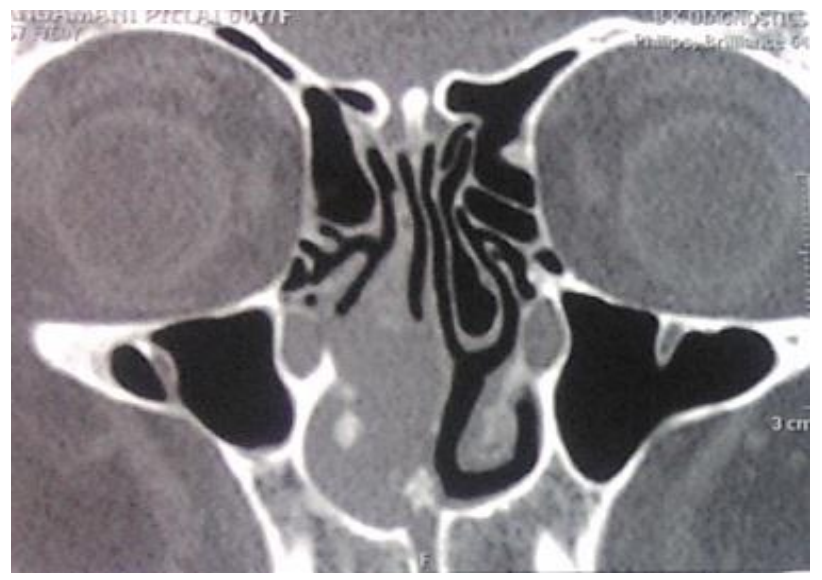

Figure 1: Prechemotharapy coronal view of nose.

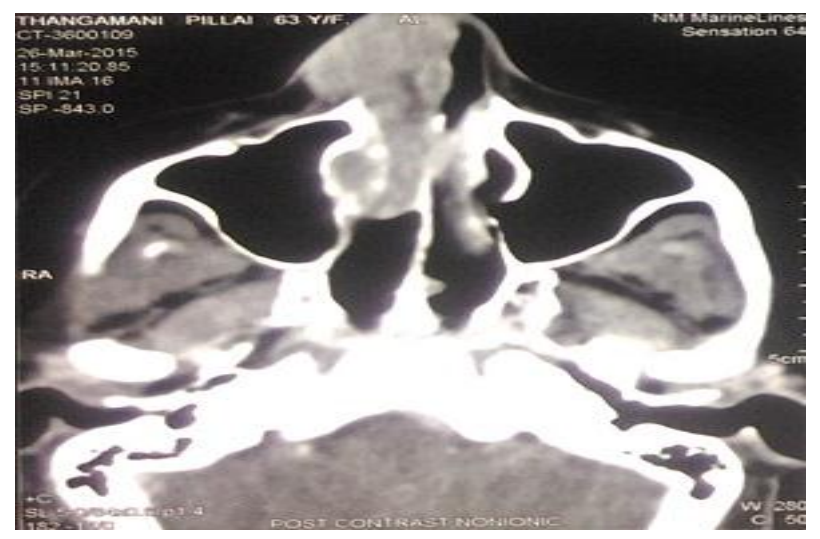

Figure 2: Prechemotherapy axial view of nose.

\section{Post chemotherapy}

After receiving chemotherapy, patient got relieved of his sign and symptoms as evident on clinical examination and follow up radiological investigations (Figures 3, 4 and 5).

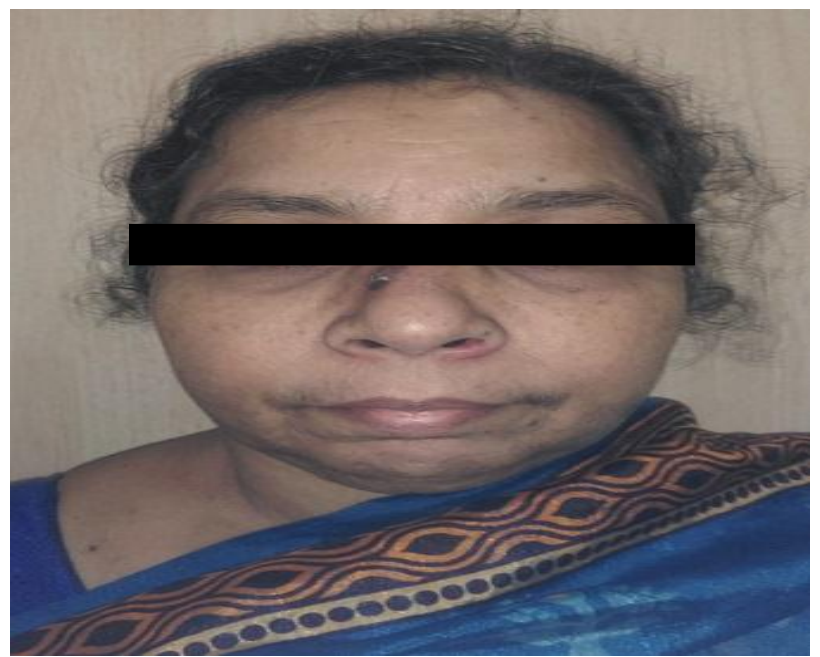

Figure 3: Front view. 


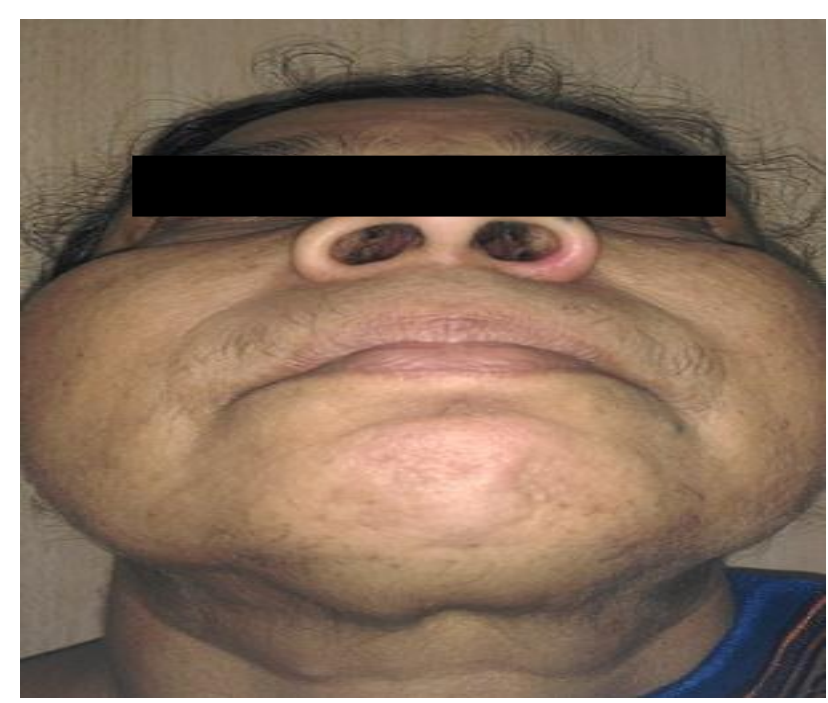

Figure 4: Basal view.

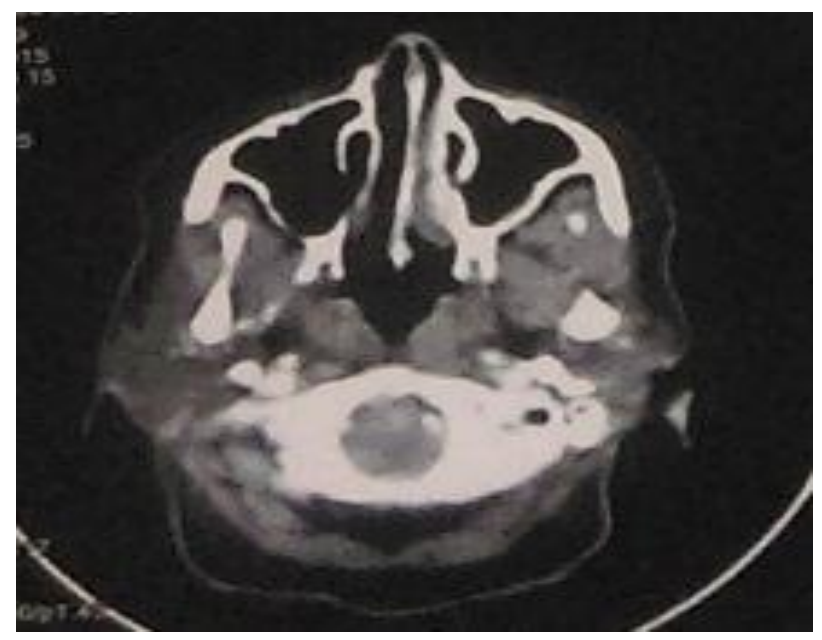

Figure 5: Axial view of nose.

\section{Second case}

A 50 years old male (Figure 1) presented in the OPD of ENT department in Dr. Babasaheb Ambedkar central railway hospital with complaints of left nasal obstruction since four years, which was insidious in onset and gradually progressive in nature. Later it progresses towards right side. It was associated with anosmia. There was no diurnal variation. Following which patient developed left side proptosis. It was insidious in onset and gradually progressive. It was associated with dryness of eyes, blurring of vision and headache. Patient later developed epiphora about ten days ago. There was no history of epistaxis, headache or decreased vision, nasal trauma or any facial pain. The consent of the patient was taken before clinical examination and taking photograph. On examination, a polypoidal growth was found in left middle meatus which did not bleed on probing. Small polypoidal growth was also seen on right side of nasal cavity. On cold spatula test, frosting was decreased on right side. Bilateral pupils were normal reacting to light.
Bells phenomenon was present on left side. Proptosis was present on left side, amounting to $1 \mathrm{~cm}$. Extraocular muscles mobility was decreased in all directions in left eye. Patient later underwent computed tomography scan of orbit and paransasal sinuses (Figure 7 and 8).

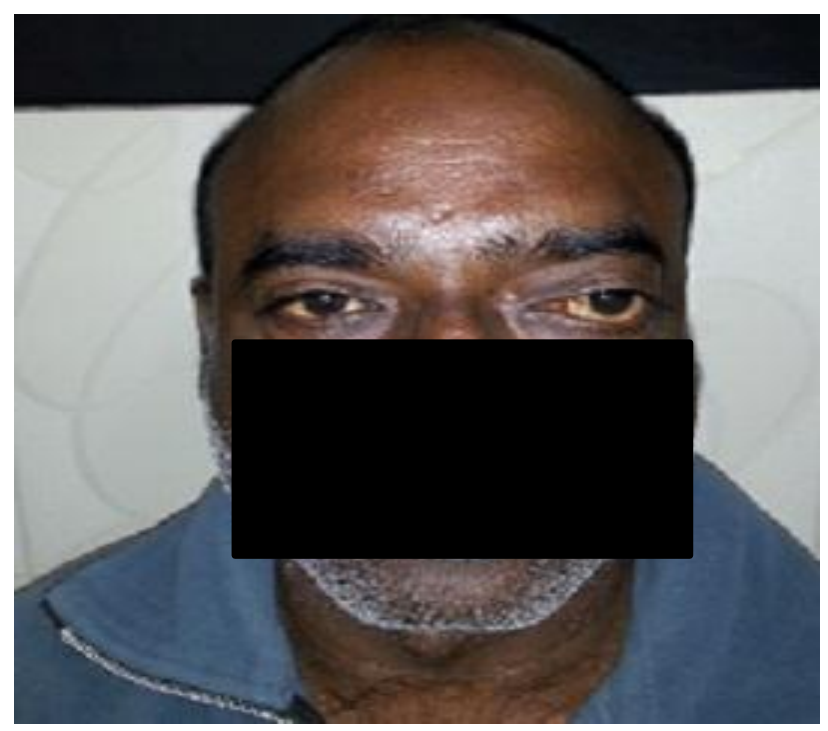

Figure 6: Proptosis in left eye.

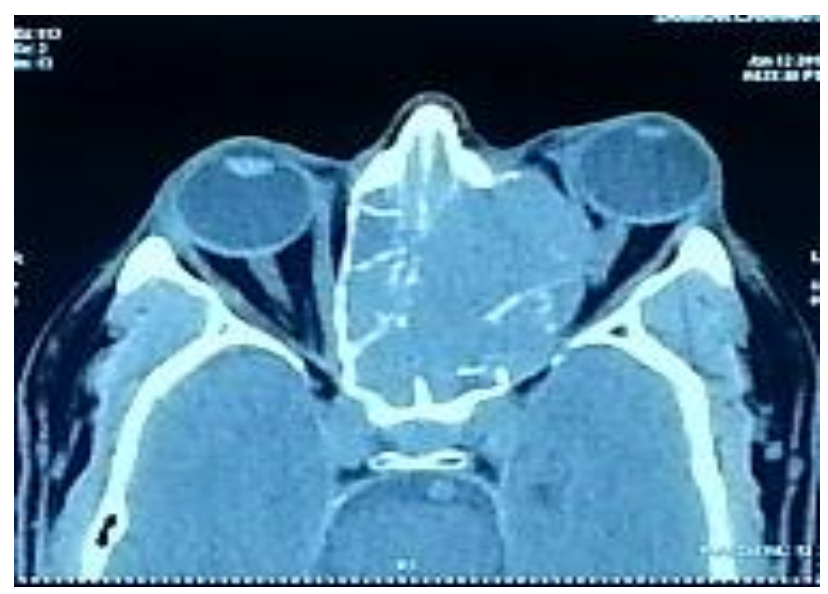

Figure 7: Axial view of nose and orbit.

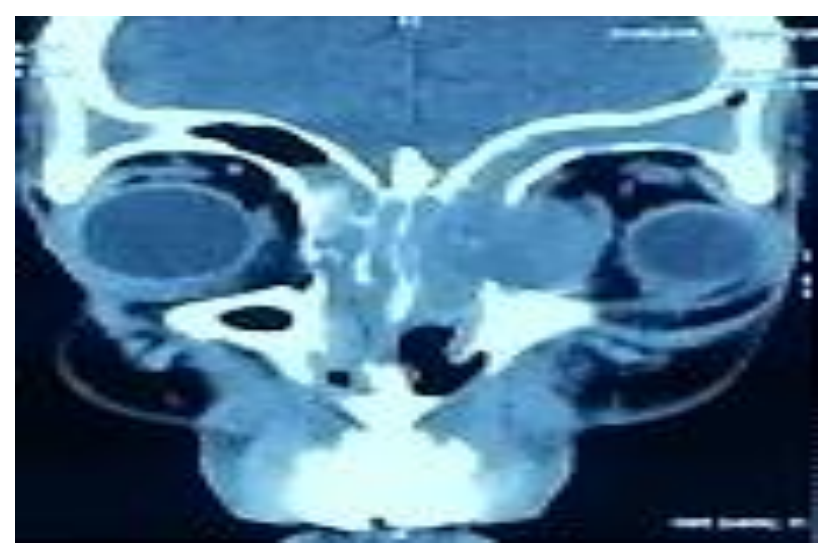

Figure 8: Coronal view of nose and orbit. 
Patient underwent right nasal mass biopsy and the specimen was sent for histopathological study. The histopathological examination reveals high grade nonHodgkin lymphoma, diffuse large B-cell type, positive for LCA, CD 20, negative for CD3, CD5, CD30, CD56, CKIT, EBV, LMPI.

\section{PET CT}

An irregular soft tissue showing subtle differential attenuation and patchy enhancement is seen in nasal cavity superiorly, causing outward bulge of ethmoid trabecular and showing mild uptake of FDG (SUV max: 6.2). No intracranial extension seen. Heterogenous enhancing lymph node with uptake SUV max: 13.8 are seen in level II of neck of left measuring $1.6 * 1.5 \mathrm{~cm}$. Few subcentimeter size nodes with only subtle uptake SUV max: 2.4 seen in bilateral Ib and right level II. No metabolically active liver/skeletal lesion is seen.

MUGA scan: LVEF-58.1\% with no RWMA.

Bone marrow biopsy: Mildly hypercellular uninvolved bone marrow.

Bone marrow aspiration: Mildly hypercellular bone marrow with $18 \%$ lymphocyte with $1 \%$ blast cells.

CSF: Negative for malignancy.

LDH: $332 \mathrm{u} / 1$

Patient later received chemotherapy comprising of injection rituximab $(700 \mathrm{mg})$, injection doxorubicin $(90$ $\mathrm{mg}$ ), injection cyclophosphamide (1400 mg), injection vincristine $(2 \mathrm{mg}$ ) for one day with injection Dexona for five days. Post chemotherapy patient got relieved from his symptoms (Figure 9).

\section{Postchemotherapy}

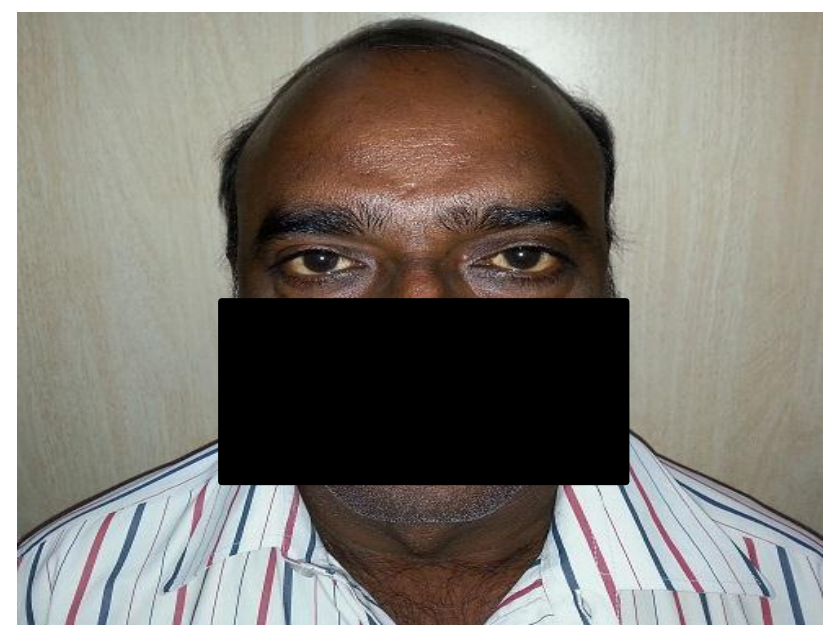

Figure 9: Proptosis relieved after chemotherapy.

\section{DISCUSSION}

Non-Hodgkin lymphoma is a diverse group of malignant lymphomas whose common feature is absence of the giant Reed-Sternberg cells characteristic of Hodgkin disease. Non-Hodgkin lymphoma consists of many different conditions which are grouped by their aggressiveness. Less aggressive forms were compatible with a long survival while more aggressive forms can be rapidly fatal without treatment. NHLs of the sinonasal tract are uncommon malignancies representing 3\%-5\% of all malignancies. ${ }^{3}$ Primary non-Hodgkin Lymphoma of the nasal cavity is a distinct clinicopathological entity. In contrast to lymph node NHL no guidelines for treatment are established for NHL of nasal cavity. Geographic factors play a role in the frequency and histological subtype of NHL of the sinonasal tract. In Asian populations and in Peru, nasal lymphomas are more common and are predominantly T-cell lymphomas, whereas B-cell subtypes are typically more common among the sinonasal lymphomas observed in Western populations. ${ }^{4}$

In a report from Poland by Zagolski et al, NHL was the most common head and neck malignancy occurring in children, and DLBCL was the most common subtype. With regard to pathology, nasal NHL is characterized by angioinvasion and necrosis. The extensive necrosis, polymorphic cellular infiltration, and small sizes of the biopsy samples usually make subtyping according to the working formulation quite difficult.

Patients with non-Hodgkin lymphoma of nasal cavity present at an early stage. However, these early stage tumors often are a bulky primary tumor, and directly extend into the neighbouring organs and tissues. The involvement of the maxillary and ethmoid sinuses is the most common mode of local extension. Conversely, the probability of lymph node enlargement and distant extranodal spread increases with extension of the primary tumor outside the nasal cavity. The major pathway of lymph node spread for NHL of the nasal cavity is to the Submandibular and cervical lymph nodes.

Lymphomas are usually sub-mucosal. They differ from squamous cell carcinomas which are usually ulcerative in appearance. Early diagnosis of primary NHL of the nasal cavity and paranasal sinuses was difficult in our patients, because this lesion develops in an anatomic space and expands toward the sinus, nasal cavity or nasopharynx, not usually causing symptoms in the early stages. Only after reaching a considerable size and involvement do the symptoms appear, and these may simulate other nasal or head and neck diseases. The most common presenting symptoms of sinonasal lymphomas are nasal obstruction, epistaxis, headache, and unilateral facial, cheek, or nasal swelling. ${ }^{6}$ Other infrequent symptoms are diplopia or blurred vision, and nasal or cheek pain. 
Our patients presented with the chief complaints of nasal obstruction with a nasal mass. We also find anosmia along with proptosis in one of our patient which suggests of the progression of the disease to the orbit and cribriform plate. Our patients did not have any lymph node involvement. Both of our patients underwent routine blood investigation and radiological examination. Patients later underwent biopsy from their nasal mass. Histopathological examination of biopsy of both the patients confirms the diagnosis.

Patients were then given chemotherapy comprising of cyclophosphamide, vincristine, doxorubicin and rituximab. Patient's symptoms get subsided after taking chemotherapy.

\section{CONCLUSION}

Non Hodgkin lymphoma of nose and paranasal sinuses is a rare presentation and difficult to diagnose due to overlapping signs and symptoms with many other nasal pathologies. Although non-Hodgkin lymphoma has a varied manner of presentation, response to therapy and prognosis, the majority of patients with localized disease can be cured with combination chemotherapy alone. It is must to do biopsy of unilateral nasal mass before any surgical intervention.

Funding: No funding sources Conflict of interest: None declared

Ethical approval: Not required

\section{REFERENCES}

1. Mitsuyasu RT. Non-AIDS defining malignancies in HIV. Top HIV Med. 2008;16:117-21.

2. Wang J, Sun N, Weinstein S, Canalis R. Primary Tcell rich B-cell lymphoma of the ethmoid sinus. A case report with 5 years of follow-up. Arch Path Lab Med. 2000;124:1213-6.

3. Quraishi M, Bessel E, Clark D, Jones N, Bradley P. Non-Hodgkin's lymphoma of the sinonasal tract. Laryngoscope. 2000;110:1489-92.

4. Longsdon M, Ha C, Kavadi V, Cabanillas F, Hess M, Cox J. Lymphoma of the nasal cavity and paranasal sinuses. Improved outcome and altered prognostic factors with combined modality therapy. Cancer. 1997;80:477-88.

5. Zagolski O, Dwivedi RC, Subramanian S, Kazi R. Non-Hodgkin's lymphoma of the sino-nasal tract in children. J Cancer Res Ther. 2010;6:5-10.

6. Vidal R, Devaney K, Ferlito A, Rinaldo A, Carbone A. Sinonasal malignant lymphomas: a distinct clinicopathological category. Ann Otol Rhinol Laryngol. 1999;108:411-9.

Cite this article as: Dalmia D, Behera SK, Bhatia JSS. Non-Hodgkin lymphoma of sinuses and nasal cavity: case series of two. Int J Otorhinolaryngol Head Neck Surg 2017;3:737-41. 